\title{
Varicella Zoster Acute Retinal Necrosis: A Case Report and Brief Review of the Literature
}

Justin Yamanuha M.D, Bharath Raj Palraj M.B.B.S, Ala S Dababneh M.D, and Olivia R Mohan D.O

Mayo Clinic Health System, Franciscan Healthcare, La Crosse, Wisconsin, US

\begin{abstract}
A 50-year-old woman with well controlled hypertension, hyperlipidemia, plaque psoriasis, exercise induced asthma, and migraine headaches presented with a few days of right eye pain. She was diagnosed with a migraine headache and referred to an Optometrist and ultimately to an Ophthalmologist. On examination, she had significant anterior and posterior uveitis, hemorrhagic retinitis, and optic nerve edema in the right eye. An aqueous aqueous fluid sample was obtained and sent for polymerase chain reaction (PCR) testing for viral deoxyribonucleic acid (DNA) and came back positive for Varicella Zoster Virus (VZV). The patient was diagnosed with Zoster acute retinal necrosis (ARN) and treated with intravitreal (intraocular) injections of Ganciclovir and Foscarnet as well as intravenous Acylovir and then oral Valacyclovir (Valtrex) and oral prednisone. ARN is a rare clinical entity occurring in healthy individuals caused most commonly by Herpes Simplex Virus 1 or 2 (HSV 1,2) or Varicella Zoster Virus (VZV) and almost always occurs without typical cutaneous dermatomal manifestations typical of Shingles. Quick identification along with aggressive antiviral and anti-inflammatory treatment can slow progression of this vision threatening disease and reduce risk of blindness and or involvement of the fellow eye.
\end{abstract}

Keywords: Acute retinal necrosis; Varicella zoster virus; Intravitreal ganciclovir; Foscarnet valacyclovir; Acyclovir

\section{Introduction}

We present a case of a generally healthy 50 -year-old female who presented with a few days of pain and redness of the right eye. She was diagnosed with a migraine headache but upon follow up with an Optometrist and then an Ophthalmologist, was identified as having significant intraocular inflammation, hemorrhagic retinitis and optic nerve edema. Work up included laboratory studies as well as an aqueous fluid sample which detected Varicella Zoster Virus (VZV) deoxyribonucleic acid (DNA) via polymerase chain reaction (PCR). The patient was diagnosed with Zoster Acute Retinal Necrosis and treated with intravitreal (intraocular) injections of Ganciclovir and Foscarnet as well as intravenous Acyclovir and oral prednisone. There was initial clinical worsening but finally stability and the patient was transitioned to oral Valacyclovir and tapered off the oral prednisone. After two months of systemic antiviral therapy, there was no retinal detachment in the affected right eye and no involvement of the left eye. ARN is a rare clinical entity but is a severe form of ocular inflammation and almost always presents in the absence of simultaneous clinical cutaneous findings zoster or simplex rashes in healthy individuals.

\section{Materials and Methods}

This article represents a single patient case report and brief review of the literature on acute retinal necrosis, anti-viral therapy for Zoster, and Zoster vaccine administration.

\section{History}

A 50-year old healthy woman described right eye pain with eye movement and photophobia for a few days. She was seen at an urgent care facility, had a negative non-contrast computerized tomography (CT) of the head, and was given intravenous ketorolac (Toradol) for a presumed migraine headache. The patient was asked to follow up with the eye clinic in a few days. She was initially evaluated by an Optometrist who identified anterior uveitis but suspected inflammatory demyelinating optic neuritis by history, set up a referral to an Ophthalmologist and Neurologist, and requested a Magnetic Resonance Imaging (MRI) of the brain.

Additional medical history was significant for well controlled hypertension, hyperlipidemia, plaque psoriasis, mild exercise induced asthma, gastroesophageal reflux disease (GERD) and occasional migraine headaches. The patient did have chickenpox as a child. There were no new medications, recent travel or new sexual partners. Family history was positive for a son who lost vision from a mitochondrial condition called Leber's Hereditary Optic Neuropathy (LHON). Review of systems was negative for chest pain, new rashes, oral or genital ulcers, joint pains, fevers or chills, weight loss or night sweats.

\section{Examination}

Dilated eye examination by the Ophthalmologist identified anterior and posterior uveitis, inflammatory hemorrhagic retinitis and optic disc edema (Figure 1). Retinal vascular fluorescein angiography (FA) and optical coherence tomography (OCT) were obtained confirming active retinal vascular and optic nerve inflammation (Figures 2 and 3). The left eye was asymptomatic and free of ocular inflammation or other pathology. Differential diagnosis included non-infectious causes of panuevitis including Sarcoidosis and Wegener's Granulomatosis as well as infectious causes of panuveitis including Tuberculosis, Syphilis, Toxoplasmosis, and bacterial endophthalmitis. Viral etiologies including Herpes Simplex or Varicella Zoster related acute retinal necrosis (ARN) or progressive outer retinal necrosis (PORN), or

*Corresponding author: Justin Yamanuha, Medical Retina Specialist Consultant Mayo Clinic Health System Franciscan Healthcare, La Crosse, Wisconsin Assistant Professor of Ophthalmology, Mayo Clinic, Rochester, Minnesota, USA, Tel: 6083929871; Fax: 6083929360; E-mail: Yamanuha.justin@mayo.edu

Received June 04, 2017; Accepted June 10, 2017; Published June 15, 2017

Citation: Yamanuha J, Palraj BR, Dababneh AS, Mohan OR (2017) Varicella Zoster Acute Retinal Necrosis: A Case Report and Brief Review of the Literature. Immunochem Immunopathol 3: 126. doi: 10.4172/2469-9756.1000126

Copyright: @ 2017 Yamanuha J, et al. This is an open-access article distributed under the terms of the Creative Commons Attribution License, which permits unrestricted use, distribution, and reproduction in any medium, provided the original author and source are credited. 
Citation: Yamanuha J, Palraj BR, Dababneh AS, Mohan OR (2017) Varicella Zoster Acute Retinal Necrosis: A Case Report and Brief Review of the Literature. Immunochem Immunopathol 3: 126. doi: 10.4172/2469-9756.1000126

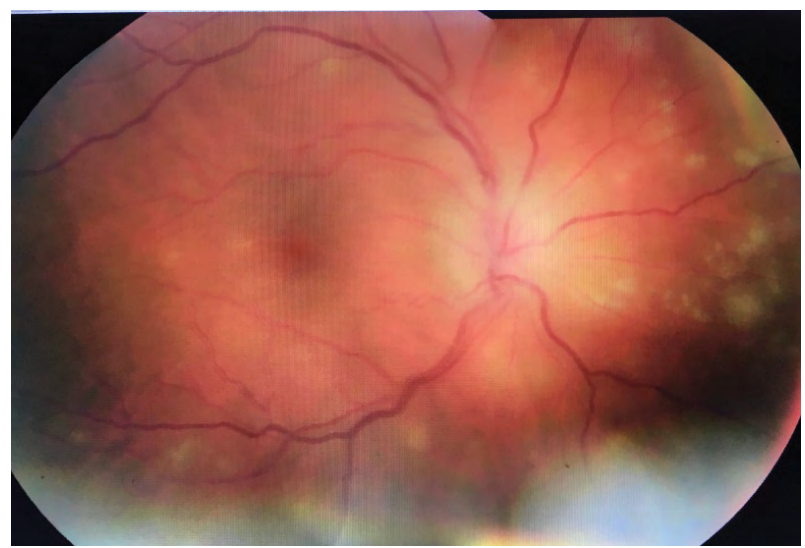

Figure 1: Montage fundus photo of the right eye demonstrating multifocal areas of retinitis as well as optic disc edema.

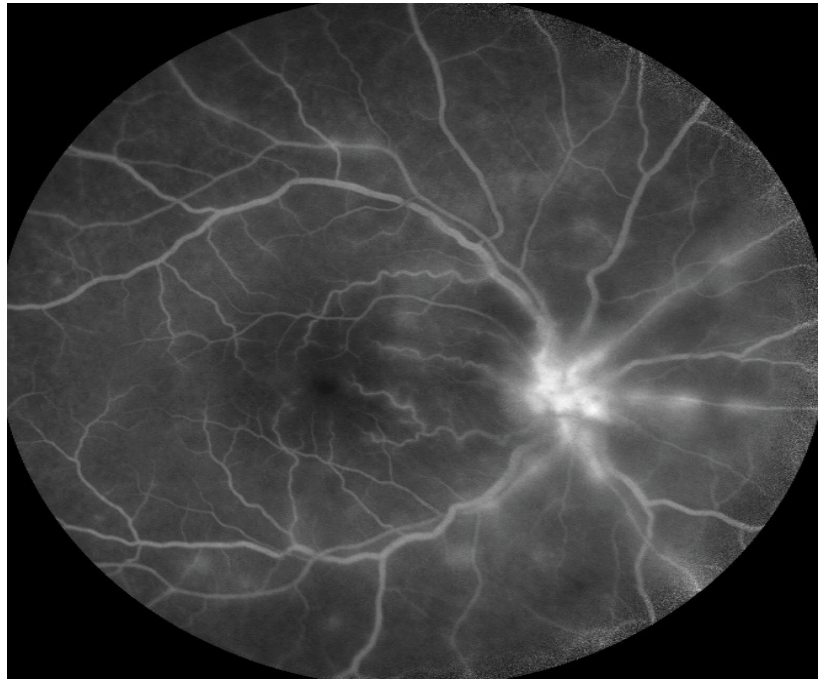

Figure 2: Fluorescein angiogram of the right eye demonstrating multiple foci of retinal vascular inflammation as well as optic disc leakage.

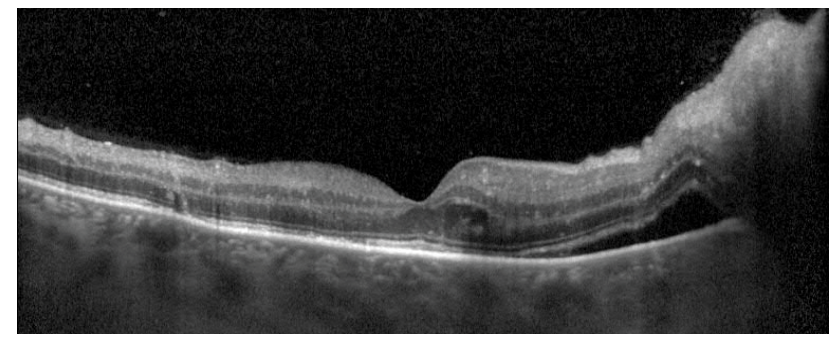

Figure 3: Ocular coherence tomography (OCT) of the right eye demonstrating inflammation of the optic nerve and macula.

cytomegalovirus (CMV) retinitis. Immune suppression was unknown but seemed unlikely in this case, making PORN or CMV retinitis less likely, as these tend to occur in immune suppressed individuals.

\section{Diagnosis and management}

A diagnostic anterior chamber aqueous fluid specimen was obtained from the right eye and sent for DNA analysis via PCR for the common causes of viral retinitis (Herpes Simplex Virus 1,2, Varicella
Zoster Virus, and Cytomegalovirus). Simultaneously, an intravitreal injection of Ganciclovir $2 \mathrm{mg}$ in $0.1 \mathrm{~mL}$ was injected into the right eye to treat these potential viral etiologies. Laboratory studies for complete blood count, Tuberculosis, Lyme disease, Syphilis, Sarcoidosis, and Wegener's Granulomatosis were ordered and oral Valacyclovir (Valtrex 1 gram three times daily) was given as an outpatient prescription.

\section{Clinical course}

The patient returned two days later with increased inflammation and retinitis in the right eye (Figure 4). The PCR revealed Varicella Zoster Virus DNA. An intravitreal injection of Foscarnet $2.4 \mathrm{mg} / 0.1$ $\mathrm{mL}$ was given in the right eye and the Infectious Disease Service was consulted. The patient had a peripherally inserted cutaneous catheter (PICC) line placed and was started on intravenous Acyclovir $10 \mathrm{mg} /$ $\mathrm{kg}$ divided into every 8 hour doses. Oral prednisone $40 \mathrm{mg}$ per day was added to help reduce the inflammatory burden along with a topical steroid eye drop four times daily.

Over the next few days there was slow progression and ultimately stabilization of the Zoster acute retinal necrosis. The patient tolerated two weeks of intravenous acyclovir without any adverse effect and was transitioned back to oral Valacylovir 1 gram three times daily. Adequate hydration and avoidance of concomitant nephrotoxic drugs were ensured to minimize risk of renal injury and serial weekly creatinine monitoring did not detect any signs of nephrotoxicity. One additional intravitreal Ganciclovir $2 \mathrm{mg}$ in $0.1 \mathrm{~mL}$ was given in the second week of treatment. After eight weeks, there was persistent vitreous haze but stabilization of the retinal necrosis without development of retinal detachment in the right eye (Figure 5), nor involvement of the left eye. Oral prednisone was tapered and then discontinued and the patient was transitioned from treatment dose Valacylovir to suppressive dose 1 gram daily to be continued for several months. A topical steroid eye drop was needed for months for residual ocular inflammation. There was no progression of cataract or elevation of the intraocular pressure with the long-term steroid eye drop course.

\section{Discussion}

Acute Retinal Necrosis is an uncommon but very aggressive form of potentially blinding ocular inflammatory disease. It typically occurs in young to middle aged healthy adults and progresses rapidly, likely due to a competent and active immune response. Patients typically present with eye pain, photophobia and vision loss and the diagnosis may be missed if a dilated eye examination is not performed. Examination findings typically include significant anterior and posterior segment intraocular inflammation as well as typical foci of hemorrhagic retinitis. The differential diagnosis for ARN includes Cytomegalovirus (CMV) retinitis, Toxoplasma Chorioretintis, Tuberculous or Syphilitic Choroiditis as well as atypical vascular occlusions.

The most typical causative organisms are members of the Herpes viridae family including Herpes Simplex Virus (HSV) [1,2] and Varicella Zoster Virus (VZV). Most typically younger patients are found to have ARN caused by HSV-2 whereas older patients tend to have ARN secondary to HSV-1 or VZV $[1,2]$. There are known associations with prior clinical cutaneous Zoster infections or Herpes Simplex Encephalitis or neonatal herpes virus infections [2]. The distinction between these possible etiologies is most typically made by intraocular fluid sampling (aqueous or vitreous) for viral DNA detectable by PCR $[1,2]$. Serologic testing for these nearly ubiquitous organisms is less critical for diagnosis and presumptive treatment of ARN should not be delayed while waiting for return of diagnostic test results. 


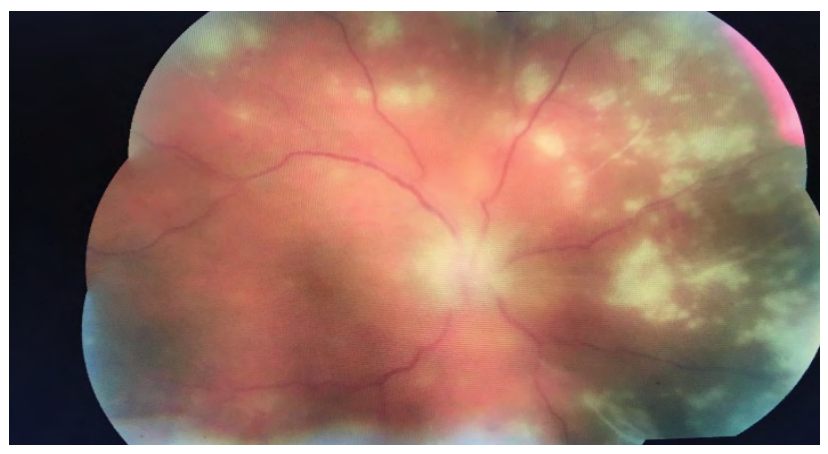

Figure 4: Fundus photo of the right eye two days after initial presentation and injection of intravitreal ganciclovir showing initial worsening of the acute retinal necrosis in the right eye.

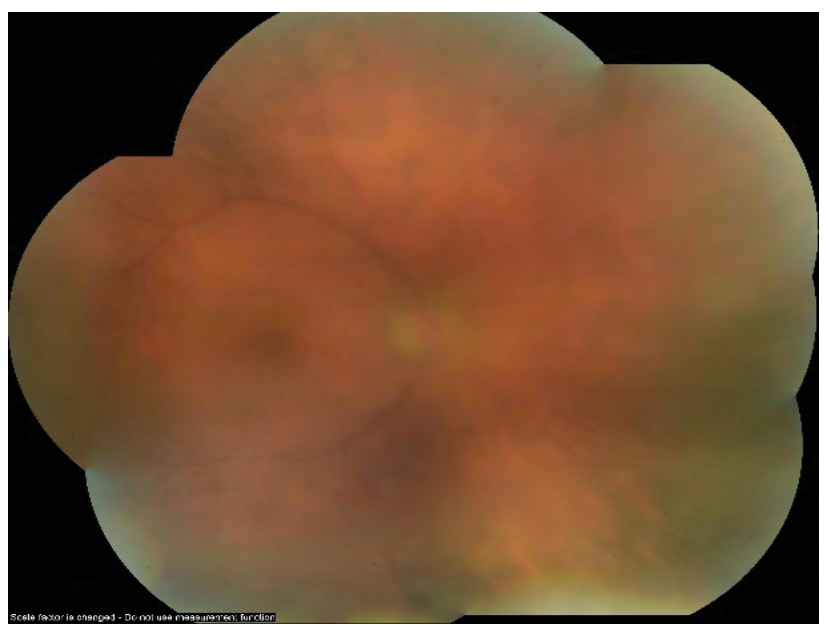

Figure 5: Fundus photo of the right eye two months after presentation showing focal areas of retinal fibrosis in the periphery without retinal detachment.

As with other rare diseases, there are no randomized clinical trials to guide the optimal management in these cases. Treatment of ARN typically involves local intravitreal antiviral injections of Ganciclovir 2 $\mathrm{mg} / 0.1 \mathrm{~mL}$ and or Foscarnet $2.4 \mathrm{mg} / 0.1 \mathrm{~mL}$. Systemic antiviral therapy with intravenous Acyclovir $5-15 \mathrm{mg} / \mathrm{kg} / \mathrm{day}$ or oral Valacyclovir 1000 mg three times daily for an induction phase (typically one to two weeks), followed by oral Valacyclovir $1000 \mathrm{mg}$ three times daily for at least 4-6 more weeks (a total of 6- 12 weeks of high dose systemic antiviral therapy) $[1,2]$. Intravenous acyclovir may cause crystalline nephropathy, so adequate hydration and monitoring of renal function during this induction phase with high dose systemic antiviral therapy is paramount. Abrupt cessation of therapy in cases of crystalline nephropathy secondary to acyclovir can often reverse toxicity and limit permanent damage [3]. In an immunocompetent host, maintenance antiviral therapy can be recommended for three to six months, based on expert opinion, and sometimes indefinitely if patients have recurrences or reactivation of ARN.

The primary goal of the combination local ocular and systemic approach is to reduce viral replication in the affected eye and also reduce the risk of involvement of the second eye. The use of aspirin to reduce retinal vascular thrombosis is not uniformly recommended, but topical corticosteroid eye drops as well as oral prednisone are usually encouraged to reduce the heavy inflammatory burden. The ultimate visual prognosis depends on the degree of retinal involvement as large areas of retinitis can lead to retinal detachments which are difficult to repair as these typically involve thin, necrotic retina. Early diagnosis and aggressive treatment preserves vision.

The natural history of Herpes viruses in humans is primary infection (e.g., chicken pox from Varicella) followed by latency. For Varicella, the dorsal root ganglion is the primary site of latency and reactivation typically manifests as dermatomal Zoster associated with a particular dorsal nerve root during periods of high stress, immune senescence, or immunodeficiency. A critical distinction between ARN and the more commonly occurring and identified dermatomal Herpes Zoster Ophthalmicus (HZO) is that Acute Retinal Necrosis most typically occurs in the absence of concurrent dermatomal HZO. ARN is not dermatomal Zoster, but rather it is suspected that prior exposure to varicella either as primary chicken pox infection or secondary Shingles Zoster is most typical, such that ARN occurs from hematogenous spread $[4,5]$.

It is also most typically the case that patients with ARN do not have active systemic viremia. There is a report of two immunocompromised patients who developed cutaneous HZO on one side of the face and then developed acute retinal necrosis in the fellow eye [6]. The authors propose that the eyes of the patients who developed HZO were able to mount a protective immune response protecting them from the more severe posterior acute retinal necrosis which affected the fellow eyes not affected by HZO.

As the aging population continues to increase, the number of adults with Zoster and administration of the Zoster vaccine will likely continue to increase. In 2014, $28 \%$ of adults in the United Stated over age 60 self-reported Shingles vaccination, which was increased from the prior reporting survey from 2013 [7]. The United States Centers for Disease Control reports that administration of the Zoster Vaccine reduces the risk of clinical Shingles in adults by $50 \%$ [8]. There is no contraindication to Zoster administration in patients with prior Shingles or ARN and it is encouraged in patients with Zoster as one event does not protect against a subsequent episode. There have been reports of patients developing Zoster ARN after Zoster vaccine administration $[9,10]$. The three patients in these two reports were all relatively immune suppressed. Two of the patients developed Zoster ARN after Zostavax [9] and one patient developed Zoster ARN after Varivax [10]. It is important that Primary Care Providers understand that this clinical phenotype can occur after vaccination, as they will be overseeing administration of these vaccines in children and adults. Special surveillance is needed in those who have had prior ARN or Shingles a period

ARN is a rare clinical phenomenon that typically occurs in healthy adults and can progress rapidly without quick identification and treatment. Diagnostic evaluation includes intraocular fluid sampling for viral DNA via PCR and treatment involves intraocular antiviral injections, systemic antiviral treatment and often steroid eye drops and systemic corticosteroids. Close monitoring is paramount as these patients may develop retinal detachments or involvement of their fellow eyes. It is unclear if these patients should receive subsequent Zoster vaccination, but as during the active phase of the disease, close monitoring is important as reactivation ARN could possibly occur and any suppressive antiviral therapy needs to be halted or adjusted for a few weeks prior to and sometimes after vaccination in order to ensure adequate immunologic protection from the Zoster vaccination administration [8]. 
Citation: Yamanuha J, Palraj BR, Dababneh AS, Mohan OR (2017) Varicella Zoster Acute Retinal Necrosis: A Case Report and Brief Review of the Literature. Immunochem Immunopathol 3: 126. doi: 10.4172/2469-9756.1000126

Page 4 of 4

\section{References}

1. Taney L, Adam M, Thau A, Sha VA (2014) Acute Retinal Necrosis, American Academy of Ophthalmology Eye.

2. Davis JL, Van Gelder RN (2015) Necrotizing Herpetic Retinopathies: Clinicians' Corner. American Academy of Ophthalmology Focal Points.

3. Perazella MA (2017) Crystal-induced acute kidney injury (acute renal failure).

4. Gnann JW (2002) Varicella-zoster virus: atypical presentations and unusual complications. Journal of Infectious Diseases 186: S91-S98.

5. Gnann JW, Whitley RJ (2002) Herpes Zoster. New England Journal of Medicine 347: 340-346.

6. Matthews BN, Erb N, Gordon C, Callear AB, Murray PI, et al. (2002) Unilateral varicella zoster virus ophthalmicus and contralateral acute retinal necrosis. Eye 16: 778-780

7. United States Centers for Disease Control. Shingles (Herpes Zoster) Surveillance.

8. United States Centers for Disease Control (2017) Shingles (Herpes Zoster) Vaccine and Preventable Disease.

9. Charkoudian LD, Kaiser GM, Steinmetz RL, Srivastava SK (2011) Acute retina necrosis after herpes zoster vaccination. Archives of Ophthalmology 129 : 1495-1497.

10. Gonzales JA, Levison AL, Stewart JM, Acharya NR, Margolis TP (2012) Retinal necrosis following varicella-zoster vaccination. Archives of Ophthalmology 130 1355-1356. 